\title{
Brain Single Photon Emission Computed Tomography With Tc-99m MIBI or Tc-99m ECD in Comparison to MRI in Multiple Sclerosis
}

\author{
Majid Assadi, MD, * Hooman Salimipour, MD, $†$ Mohammad Seyedabadi, PhD, * Jamshid Saberifard, MD, + \\ Hamid Javadi, MD, $\S$ Iraj Nabipour, MD, * and Reza Nemati, MD ${ }^{*}$
}

\begin{abstract}
Purpose: To evaluate whether or not brain single photon emission computed tomography (SPECT) with Tc-99m MIBI or Tc-99m ECD (ethyl cysteinate dimer) can detect any abnormality in patients with definite multiple sclerosis (MS). We then compared these values with the results of T1, T2, and fluid-attenuated inversion recovery in magnetic resonance imaging (MRI). Materials and Methods: A total of 16 patients with proved MS were enrolled in the study, and the MRI with and without gadolinium contrast and also brain SPECT with Tc-99m MIBI ( 8 cases) or Tc-99m ECD ( 8 other cases) were performed.

Results: MRI studies was performed in 16 patients (13 women and 3 men, aged 16-38 years) and an average of 10.47, 3.7, 5.3, 1.7, and 0.9 lesions was found in respect in periventricular white matter, juxtacortical white matter, corpus callosum, cerebellar peduncles, and brainstem, whereas brain SPECT with Tc-99m MIBI or Tc-99m ECD detected no abnormality. In addition, 6 cases had some degree of contrast enhancement.

Conclusions: It seems that brain SPECT with Tc-99m MIBI or Tc-99m ECD would not improve this insufficiency. The small sizes of some plaques, particularly in chronic atrophic form of lesions, and the possibility of deeper anatomic positions of plaques can explain to some extent why the MS lesions were impossible to delineate on brain scan, although additional studies are needed.
\end{abstract}

Key Words: multiple sclerosis, brain SPECT, Tc-99m MIBI, Tc-99m ECD, magnetic resonance imaging

(Clin Nucl Med 2010;35: 682-686)

$\mathrm{D}$ emyelination of white matter (WM) in the central nervous system (CNS) is the hallmark of multiple sclerosis (MS). There has been a great amount of modifications in diagnosis of MS; although Schumacher criteria contains only clinical observations, and Poser et al have included paraclinical evaluations like cerebrospinal fluid (CSF) examination, oligoclonal banding or intrathecal IgG state, and computed tomography (CT) with little respect to magnetic resonance imaging (MRI), ${ }^{1}$ Mc Donald criteria extensively rely on MRI. Clinical evidence or imaging testimony of 2 separated lesions in CNS (dissociation in place) and/or presence of distinct lesions during a period of time (dissociation in time) are highly restricted to MS. ${ }^{2}$ Gean-Marton et al have also proposed that lesions in midsagittal

Received for publication June 11, 2009; revision accepted February 16, 2010. From the *Bushehr Research Center for Nuclear Medicine, The Persian Gulf Biomedical Sciences Institute, Bushehr University of Medical Sciences, Bushehr, Iran; Departments of $†$ Neurology, and $\$$ Radiology, Faculty of Medicine, Bushehr University of Medical Sciences, Bushehr, Iran; and §Department of Nuclear Medicine, 5th Azar Hospital, Golestan University of Medical Science, Gorgan, Iran.

Supported by Bushehr University of Medical Sciences (grant no. 145).

Reprints: Majid Assadi, MD, Bushehr Research Center for Nuclear Medicine, The

Persian Gulf Biomedical Sciences Institute, Boostan 19 Alley, Sangi Street,

Bushehr, Iran. E-mail: assadipoya@yahoo.com, asadi@bpums.ac.ir.

Copyright (C) 2010 by Lippincott Williams \& Wilkins

ISSN: 0363-9762/10/3509-0682 long repetition time (TR)/short echo time (TE) images and involvement of corpus callosum are highly specific for MS rather than other periventricular WM disorders. ${ }^{3}$ Fast spin-echo (FSE), fluid-attenuated inversion recovery (FLAIR) FSE, and short tau inversion recovery FSE are among the other modifications to MRI, which ameliorate the sensitivity and specificity of indicated MS plaques. ${ }^{4}$ A combinational set of textural features application in image volumetric interpretation has also shown to be more accurate in distinguishing MS plaques from the normal appearing $\mathrm{WM}$ and normal $\mathrm{WM}^{5}$

The most challenging concern regarding the MRI application for MS evaluation is the commonly poor correlation between MRI indicated levels of disease and clinical manifestations or expanded disability status scale in which the degree of lesions reported in MRI is always worse than what the extent of clinical symptoms are. ${ }^{6}$ Although early anticipation of potential deterioration of disease is important for clinical management, ${ }^{7}$ little prognostic capability of MRI where it cannot predict the relapse or consequent burden is another insufficiency of this procedure. ${ }^{8}$ Intracortical lesions which have been shown to be abundant in MS patients and may elicit cognitive deficit usually remains obscure on MR imaging. ${ }^{9}$

Abnormal brain scan is reported in MS and other demyelinating disorders. Gize and Mishkin have described focal abnormalities with $203 \mathrm{Hg}$-chlormerodrin or $99 \mathrm{mTcO} 4$ brain scan in $36 \%$ of cases with acute $\mathrm{MS}^{10}$ and Miller and Potsaid have also reported a case of MS with vascular infiltration in which $99 \mathrm{mTcO} 4$ scanning has revealed discrete area of hyperactivity and hyperintensity in occipital regions of the brain. ${ }^{11}$ On the other hand, Moses et al studying 14 patients in their acute exacerbation and 5 cases in their quiescent phase of the disease, who were suffering from definite MS for several years and a moderate to severe state of disability, concluded that $99 \mathrm{mTcO} 4$ scanning is unlikely to detect a state of brain abnormality in MS. ${ }^{12}$

In this study, we aim to evaluate whether or not brain SPECT scintigraphy with Tc-99m MIBI or Tc-99m ECD can detect any abnormality in patients with definite MS. We then compared these values with the results of T1, T2, and FLAIR in MRI.

\section{MATERIALS AND METHODS}

Our study population consisted of 16 patients with definite MS diagnosed on the basis of clinical signs and symptoms localized to at least 2 anatomic regions of the CNS and at least initially a course of relapses and remissions separated by at least 1 month. Each patient underwent a complete neurologic examination.

MRI was performed for all 16 cases while brain SPECT with Tc-99m ECD was performed for 8 cases and brain SPECT with Tc-99m MIBI for the other 8 subjects.

\section{Brain SPECT With Tc-99m ECD}

A commercial ECD preparation was used in 8 patients. The labeling and quality control procedures were performed according to the manufacturer's instructions. All subjects had an intravenous (IV) line established while they were lying down, with their eyes closed and ears unplugged, in a quiet darkened room with low ambient sound 
and light. After approximately 30 minutes, each subject received a $740-\mathrm{MBq}(20 \mathrm{mCi}) \mathrm{IV}$ injection of tracer while they were still lying down in the same quiet darkened room. One hour after IV injection of $740 \mathrm{MBq}(20 \mathrm{mCi}) \mathrm{Tc}-99 \mathrm{~m} \mathrm{ECD}$ in a room with low level of ambient light and minimal background noise, SPECT procedure was performed.
Scans were performed on a dual head ADAC camera (ADAC Genesys, Malpitas, CA), equipped with a pair of low energy, high resolution collimators. The full-width at half maximum (FWHM) of this system, as measured in-house, was 12-mm for Tc-99m. Standard head positioning was based on uniform alignment of the external auditory meatus
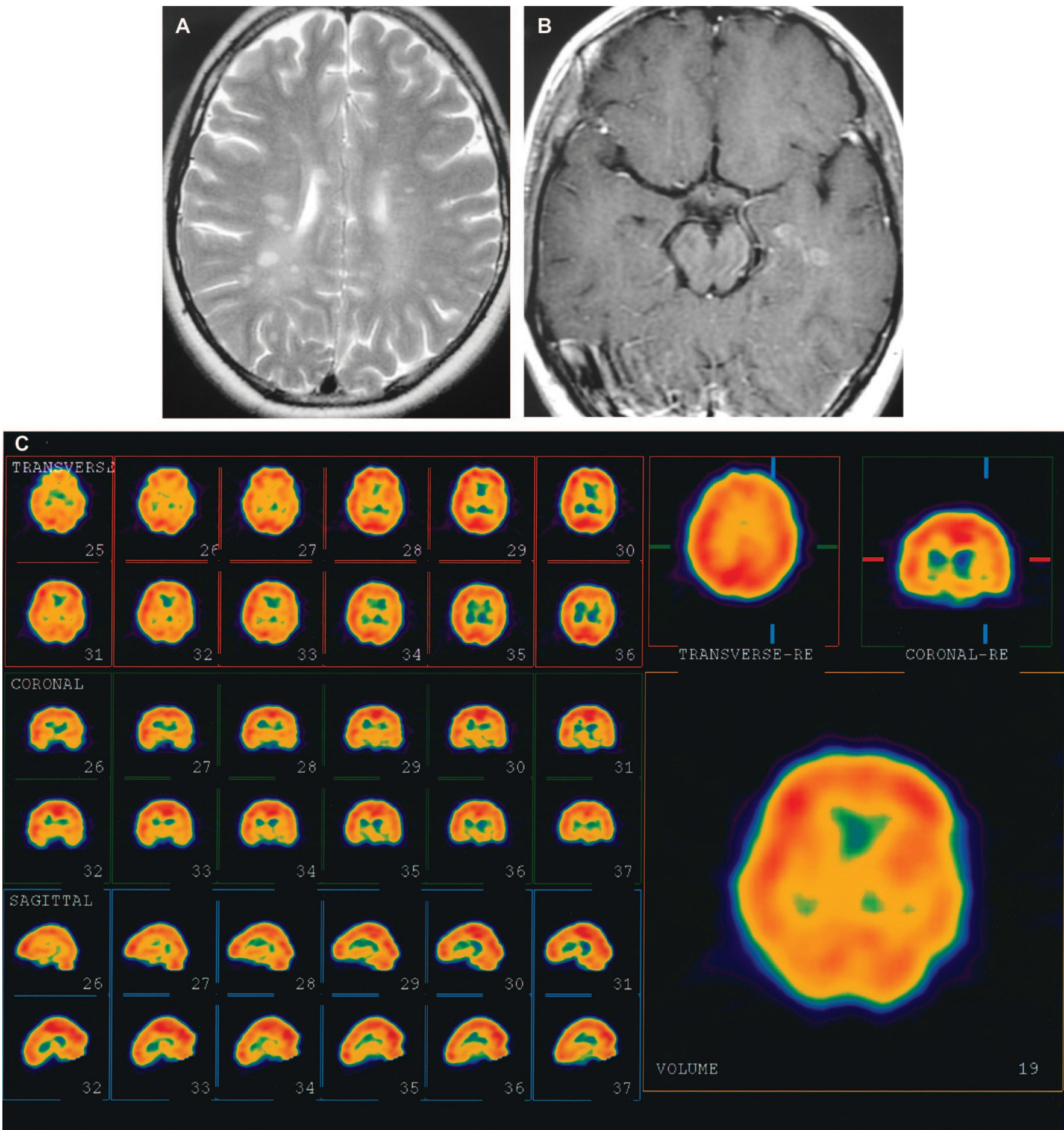

FIGURE 1. MRI and SPECT with TC-99m ECD outcomes in a 26-year-old female patient; (A): axial T2 and (B) postcontrast T1weighted images show multiple demyelinating lesions in bilateral cerebral WM, some of them showing enhancement after contrast injection. C, Brain SPECT with Tc-99m ECD shows no remarkable abnormality. 
using automated table positioning and camera-to-head-detector ratio values. The total acquisition time was 35 minutes for each study. Images were acquired in a $64 \times 64 \times 64$ three-dimensional pixel matrix at 64 steps, 30 seconds each step. Before reconstruction of the images, attenuation correction of the images was carried out by the Chang method (attenuation coefficient $0.12 \mathrm{~cm}^{-1}$ ). The data were then processed by back projection and filtered by Butterworth filter, using a
Nyquist frequency cut-off of 0.5 and order of 5. Images were reconstructed and displayed in all 3 orthogonal planes. SPECT results were analyzed by 2 experienced nuclear medicine physicians, who were not aware of the patient's medical history, nor had information about the MRI results; the frontal, parietal, temporal, occipital cortex, basal ganglia, brainstem, and cerebellum were analyzed systematically. Normal brain SPECT findings included homogeneous rCBF in the men-
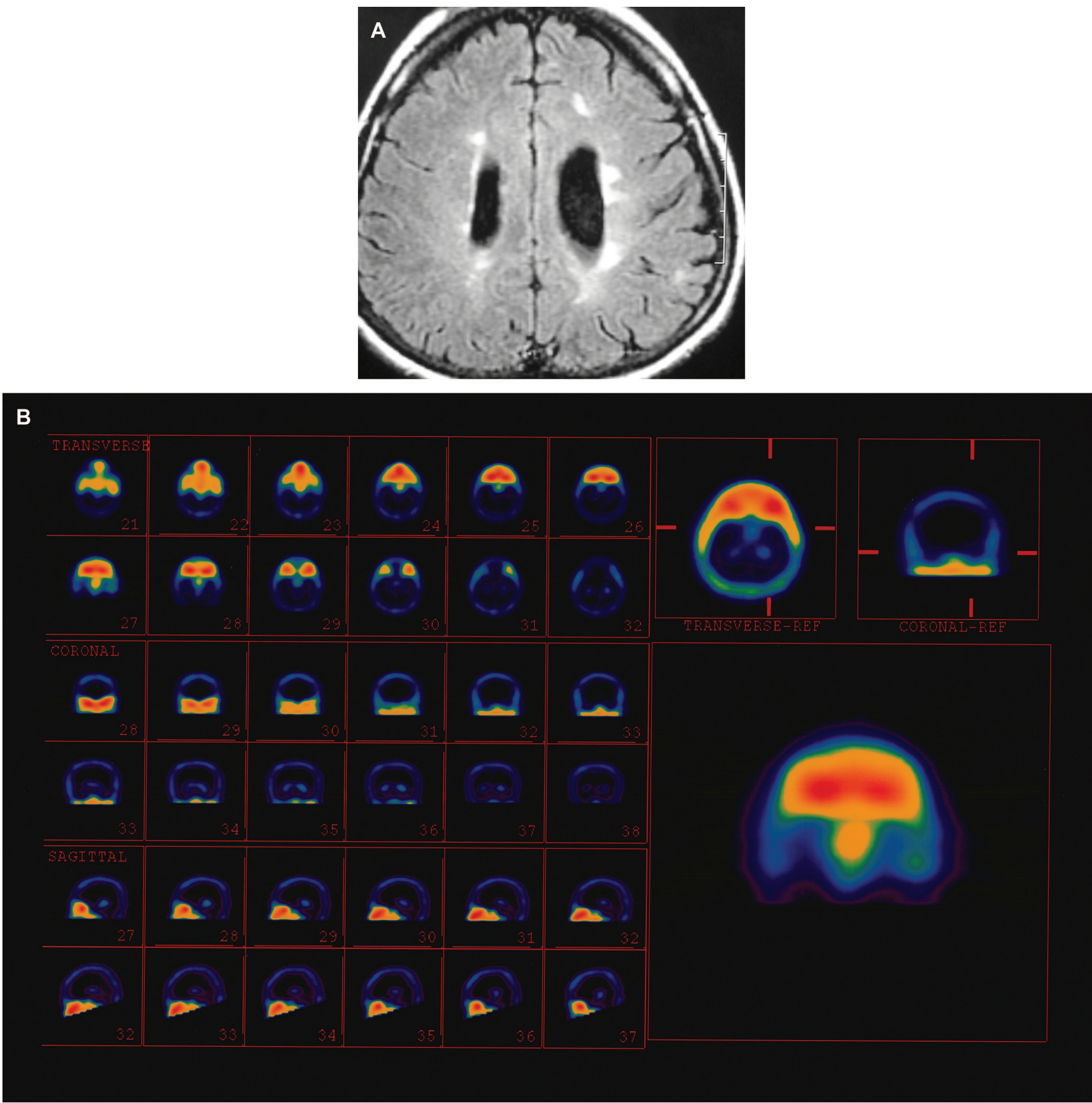

FIGURE 2. MRI and SPECT with Tc-99m MIBI outcomes in a 19-year-old woman. A, Axial FLAIR MR image shows multiple hyperintense lesions in bilateral periventricular cerebral white matter consistent with patients known multiple sclerosis. After contrast injection, no significant abnormal enhancement could be identified. B, Brain SPECT with Tc-99m MIBI shows no remarkable abnormality. 
tioned regions without focal hypoperfusion or visible asymmetry. Abnormal brain SPECT findings were defined as heterogeneous rCBF with focal hypoperfusion or visible asymmetry in at least 2 consecutive slices in 2 sections. ${ }^{13}$

\section{Brain SPECT With Tc-99m MIBI}

A commercial MIBI preparation was used in other 8 patients. The labeling and quality control procedures were performed according to the manufacturer's instructions. Each patient received 740$\mathrm{MBq}(20 \mathrm{mCi}) \mathrm{Tc}-99 \mathrm{~m} \mathrm{MIBI}$ and within 30 to 60 minutes following injection the imaging was started. All other acquisition protocol was the same as above.

\section{Magnetic Resonance Imaging}

MR imaging was performed with a 1.5-T Signal imager (General Electric, Milwaukee) with 3 spin-echo pulse sequences emphasizing T1, T2-weighted and FLAIR weighted information. We defined active lesions as those that enhanced after the IV injection of gadolinium. The scans and MRI had been performed within 1 week interval.

The study complies with the doctrine of declaration of Helsinki and was approved by the institutional ethics committee of Bushehr University of Medical Sciences. Subjects enrolled in the study were previously informed and had signed a written consent.

\section{RESULTS}

We evaluated 16 patients (13 women and 3 men, aged 16-38 years) with definite MS based on clinical signs and symptoms localized to at least 2 anatomic regions of the central nervous system and at least initially a course of relapses and remissions separated by at least 1 month. Clinical activity was defined as a recent change in neurologic signs or symptoms (within 4 weeks before the study) or a clear-cut, steady history of chronic progressive disease. All patients with diagnoses of inactive MS had been free of new neurologic symptoms for more than 4 months. Eight patients had inactive clinically.

MRI studies was performed in all 16 patients and an average of $10.47,3.7,5.3,1.7$, and 0.9 lesions was found in periventricular WM, juxtacortical WM, corpus callosum, cerebellar peduncles, and brainstem, respectively (Figs. 1A, B and 2A). Six cases had some degree of contrast enhancement.

Patients were randomly divided into 2 groups of 8 patients each and brain SPECT with either Tc-99m ECD or Tc-99m MIBI was applied. We observed abnormal brain scan neither in the former (Fig. 1C) nor in the later (Fig. 2B) assessment. Table 1 summarizes the corresponding outcomes of MRI and SPECT in each patient analyzed by 2 nuclear medicine specialists and 2 radiologists separately.

\section{DISCUSSION}

Although MR imaging provides a sensitive diagnostic tools for MS, it is noteworthy that the manifestation of plaques on MRI imaging is not specific for MS. ${ }^{2,14}$ Koziol et al have also describe that appearance of neither enhancing lesions, new enhancing lesions, nor hypointense lesions (black holes) in T1-weight MRI could anticipate the imminent clinical exacerbation of relapse-remitting MS; however, the absence of abovementioned markers in MRI is contributed to a more desirable state of the disease. ${ }^{7}$ Miki et al, using a validated highly reproducible computerized image analysis software on T2-sagittal MRI results, stated that in spite of continuous increment, the lesion volumes did not correlate with the extent of disability in relapse-remitting MS. ${ }^{15,16}$ While a moderate connection between spinal cord lesion extent and expanded disability status and sensory deficit is found in MS patients, there is inconsistency between appearance of lesions on MRI and clinical manifestations of myelopathy. ${ }^{17}$

Due to pathophysiology of MS and role of inflammation and disrupted of blood-brain barrier (BBB) and also based on the previous papers concerning of Tc-99m MIBI in these situation, we hypothesized that it may be valuable in this disease. Demyelinated regions in the brain did not result in abnormalities on none of the brain scans including brain SPECT with Tc-99m MIBI or Tc-99m ECD. Our results are somehow consistent with Moses et al study with regard to failure of brain scanning in plaques visualization; however, they have evaluated the relapse-remitting form of MS on brain scan with Tc-99m. ${ }^{12}$ The small sizes of some plaques which could be as small as $1 \mathrm{~mm}$, particularly in chronic atrophic form of lesions, and the possibility of deeper anatomic positions of plaques can to some extent explain why the $\mathrm{ms}$ lesions were impossible to delineate on brain scan $^{11,18}$; however, other investigations using SPECT modality with higher spatial resolution is required to evaluate these findings.

Gadolinium enhancement in both CT and MRI have also been applied to demonstrate a disrupted BBB in inflammatory process of MS in which the proinflammatory state of active disease and subsequent extravasation of the contrast result in both higher number of lesions detected and more intense foci. ${ }^{19,20}$ Gadolinium enhancement offers a diagnostic tool to differentiate between the active and silent states of MS and is likely to provide a superior sensitivity and specificity than CSF examination and high Iodine CT $(\mathrm{HICT})^{20,21}$; however, contrast-mediated higher intensity of foci is not confined to MS and is also observed in vasculitis as well as neurosarcoidosis and most infections ${ }^{21}$ and enhancement study may overestimate the number of active lesions. ${ }^{22}$ In addition, triple dose contrast has led not only to higher incidence of lesion detection on MRI but also a large number of false-positive lesions due to flow artifacts. $^{23,24}$ Inflammation is not present in the chronic form of MS which is deduced from the lack of contrast enhancement in persistent lesions. ${ }^{25}$ The absence of inflammatory edema in chronic form ${ }^{2}$ can be the reason behind the inefficiency of MIBI contrast enhancement in visualization of plaques in our study.

It seems that brain SPECT with Tc-99m MIBI or Tc-99m ECD would not improve this insufficiency. It is of interest to state

TABLE 1. Number of Lesions Detected in Each Anatomical Region in Different Patients

\begin{tabular}{llrrrrrrrrrrrrrrrrrrrr}
\hline & Anatomical Region & $\mathbf{1}$ & $\mathbf{2}$ & $\mathbf{3}$ & $\mathbf{4}$ & $\mathbf{5}$ & $\mathbf{6}$ & $\mathbf{7}$ & $\mathbf{8}$ & $\mathbf{9}$ & $\mathbf{1 0}$ & $\mathbf{1 1}$ & $\mathbf{1 2}$ & $\mathbf{1 3}$ & $\mathbf{1 4}$ & $\mathbf{1 5}$ & $\mathbf{1 6}$ & Total \\
\hline MRI & Periventricular WM & 13 & 26 & 0 & 2 & 26 & 4 & 3 & 10 & 15 & 24 & 5 & 14 & 12 & 6 & 6 & 4 & 170 \\
& Juxtacortical WM & 3 & 5 & 1 & 0 & 8 & 4 & 2 & 4 & 4 & 7 & 0 & 8 & 6 & 2 & 2 & 2 & 58 \\
& Corpus callosum & 5 & 8 & 1 & 2 & 13 & 5 & 4 & 8 & 3 & 12 & 2 & 4 & 5 & 2 & 6 & 4 & 84 \\
Cerebellar peduncles & 2 & 2 & 0 & 1 & 3 & 1 & 1 & 4 & 0 & 2 & 0 & 1 & 3 & 1 & 4 & 2 & 27 \\
Brainstem & 0 & 0 & 0 & 0 & 2 & 2 & 1 & 2 & 0 & 1 & 0 & 2 & 1 & 0 & 2 & 0 & 13
\end{tabular}

MIBI

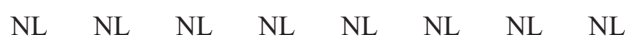

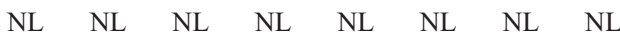

WM indicates white matter; NL, no lesion; ECD, ethyl cysteinate dimer. 
that other techniques at the field of nuclear medicine are promising regarding the evaluation of MS and stage designation of the disease; positron emission tomography (PET) of peripheral benzodiazepine receptor (PBR) with 11C-PK11195 radionucleotide had revealed that this receptors is down-regulated at chronic disappearance of myelin and noninflammatory microglia stimulation in chronic degenerative phase and myelin replacement by axonal scars, whereas PBR is up-regulated whenever there is an active stage of the disease or a relapse is impending. ${ }^{26}$ Proton MR assessment of the N-acetylaspartate which reflects direct axonal loss diminishes in MS and its dynamics may profit clinicians not only in early estimation of disease course and severity, but also in treatment decisions and patient follow up. ${ }^{8}$ Magnetization transfer (MT) imaging seems to be more specific for myelin where the MT ratio is directly proportional to myelin molecules; a decrement in MT ratio represents a structural or pathologic myelin loss and tissue injury, whereas an increment in this value indicates either an active inflammatory process or remyelination status. ${ }^{18,27}$ Average water diffusion coefficient is shown to be capable of distinguishing between relapsing-remitting and secondary-progressive lesions and is well correlated with the extent of standardized measure of disability in individual MS subjects. ${ }^{28}$

Connective tissue disease is considered one of the more important differential diagnosis of MS, which even after multiple diagnostic studies, including MRI, CSF examination, visual evoked potentials, and autoantibody marker, a number of patients with systemic autoimmune disease can be misdiagnosed for MS. ${ }^{29}$ Several studies have revealed hypoperfusion area in brain SPECT in connective tissue disease with neurologic manifestation, like systemic lupus erythematous, ${ }^{30}$ Behcet disease, ${ }^{31}$ Sjoegren syndrome, ${ }^{31}$ and antiphospholipid syndrome. ${ }^{32}$ With regard to our findings about the absence of SPECT abnormalities in MS patients, it seem that brain SPECT might be useful in discrimination of MS from connective tissue disorder, base on the evidence that hypoperfusion in SPECT is against the diagnosis of MS, although normal SPECT does not support it.

\section{CONCLUSIONS}

It seems that brain SPECT with Tc-99m MIBI or Tc-99m ECD would not improve this insufficiency. The small sizes of some plaques, particularly in chronic atrophic form of lesions, and the possibility of deeper anatomic positions of plaques can to some extent explain why the MS lesions were impossible to delineate on brain scan. It seems that brain SPECT might be useful in discrimination of MS from connective tissue disorder, based on the evidence that hypoperfusion in SPECT is against the diagnosis of MS, although normal SPECT does not support it.

\section{ACKNOWLEDGMENTS}

The authors thank the colleagues at their institutes for technical help and data acquisition.

\section{REFERENCES}

1. Poser CM, Paty DW, Scheinberg L, et al. New diagnostic criteria for multiple sclerosis: guidelines for research protocols. Ann Neurol. 1983;13:227-231.

2. Inglese M. Multiple sclerosis: new insights and trends. Am J Neuroradiol. 2006;27:954-957.

3. Gean-Marton AD, Vezina LG, Marton KI, et al. Abnormal corpus callosum: a sensitive and specific indicator of multiple sclerosis. Radiology. 1991;180: 215-221.

4. Hittmair K, Mallek R, Prayer D, et al. Spinal cord lesions in patients with multiple sclerosis: comparison of MR pulse sequences. Am J Neuroradiol. 1996; 17:1555-1565.

5. Zhang J, Tong L, Wang L, et al. Texture analysis of multiple sclerosis: a comparative study. Magn Reson Imaging. 2008;26:1160-1166.

6. McFarland HF. Correlation between MR and clinical findings of disease activity in multiple sclerosis. Am J Neuroradiol. 1999;20:1777-1778.
7. Koziol JA, Wagner S, Sobel DF, et al. Predictive value of lesions for relapses in relapsing-remitting multiple sclerosis. Am J Neuroradiol. 2001;22:284-291

8. Gonen O, Moriarty DM, Li BS, et al. Relapsing-remitting multiple sclerosis and whole-brain N-acetylaspartate measurement: evidence for different clinical cohorts initial observations. Radiology. 2002;225:261-268.

9. Geurts JJ, Bo L, Pouwels PJ, et al. Cortical lesions in multiple sclerosis: combined postmortem MR imaging and histopathology. Am J Neuroradiol. 2005;26:572-577

10. Gize RW, Mishkin FS. Brain scans in multiple sclerosis. Radiology. 1970; 97:297-299.

11. Miller SW, Potsaid MS. Focal brain scan abnormalities in multiple sclerosis. J Nucl Med. 1974;15:131-133.

12. Moses DC, Davis LE, Wagner HN Jr. Brain scanning with $99 \mathrm{~m} \mathrm{TcO} 4$-in multiple sclerosis. J Nucl Med. 1972;13:847-848.

13. Kao CH, Lan JL, ChangLai SP, et al. The role of FDG-PET, HMPAO-SPET and MRI in the detection of brain involvement in patients with systemic lupus erythematosus. Eur J Nucl Med. 1999;26:129-134.

14. Quint DJ. Multiple sclerosis and imaging of the corpus callosum. Radiology. 1991;180:15-17

15. Miki Y, Grossman RI, Udupa JK, et al. Computer-assisted quantitation of enhancing lesions in multiple sclerosis: correlation with clinical classification. Am J Neuroradiol. 1997;18:705-710.

16. Miki Y, Grossman RI, Udupa JK, et al. Relapsing-remitting multiple sclerosis: longitudinal analysis of MR images - lack of correlation between changes in T2 lesion volume and clinical findings. Radiology. 1999;213:395-399.

17. Trop I, Bourgouin PM, Lapierre Y, et al. Multiple sclerosis of the spinal cord: diagnosis and follow-up with contrast-enhanced MR and correlation with clinical activity. Am J Neuroradiol. 1998;19:1025-1033.

18. Ge Y. Multiple sclerosis: the role of MR imaging. Am J Neuroradiol. 2006;27:1165-1176.

19. Grossman RI, Braffman BH, Brorson JR, et al. Multiple sclerosis: serial study of gadolinium-enhanced MR imaging. Radiology. 1988;169:117-122.

20. Grossman RI, Gonzalez-Scarano F, Atlas SW, et al. Multiple sclerosis gadolinium enhancement in MR imaging. Radiology. 1986;161:721-725.

21. Tas MW, Barkhol F, van Walderveen MA, et al. The effect of gadolinium on the sensitivity and specificity of MR in the initial diagnosis of multiple sclerosis. Am J Neuroradiol. 1995;16:259-264.

22. Bozzao A, Bastianello S, Ferone E, et al. Enhanced and unenhanced MR with magnetization transfer in multiple sclerosis. Am J Neuroradiol. 1996;17: 1837-1842.

23. van Waesberghe JH, Castelijns JA, Roser W, et al. Single-dose gadolinium with magnetization transfer versus triple-dose gadolinium in the MR detection of multiple sclerosis lesions. Am J Neuroradiol. 1997;18:1279-1285

24. Bastianello S, Gasperini C, Paolillo A, et al. Sensitivity of enhanced MR in multiple sclerosis: effects of contrast dose and magnetization transfer contrast. Am J Neuroradiol. 1998;19:1863-1867.

25. Guttmann CR, Ahn SS, Hsu L, et al. The evolution of multiple sclerosis lesions on serial MR. Am J Neuroradiol. 1995;16:1481-1491.

26. Debruyne JC, Versijpt J, Van Laere KJ, et al. PET visualization of microglia in multiple sclerosis patients using [11C]PK11195. Eur J Neurol. 2003;10: 257-264.

27. Brochet B, Dousset V. Pathological correlates of magnetization transfer imaging abnormalities in animal models and humans with multiple sclerosis Neurology. 1999;53(5 suppl 3):S12-S17.

28. Castriota Scanderbeg A, Tomaiuolo F, Sabatini U, et al. Demyelinating plaques in relapsing-remitting and secondary-progressive multiple sclerosis: assessment with diffusion MR imaging. Am J Neuroradiol. 2000;21:862-868.

29. Cikes N, Bosnic D, Sentic M. Non-MS autoimmune demyelination. Clin Neurol Neurosurg. 2008;110:905-912.

30. Chen JJ, Yen RF, Kao A, et al. Abnormal regional cerebral blood flow found by technetium-99m ethyl cysteinate dimer brain single photon emission computed tomography in systemic lupus erythematosus patients with normal brain MRI findings. Clin Rheumatol. 2002;21:516-519.

31. Kao CH, Lan JL, ChangLai SP, et al. Technetium-99m-HMPAO SPECT and MRI of brain in patients with neuro-Behcet's syndrome. $\mathrm{J} \mathrm{Nucl} \mathrm{Med}$. 1998;39:1707-1710.

32. Kao CH, Lan JL, Hsieh JF, et al. Evaluation of regional cerebral blood flow with $99 \mathrm{mTc}-\mathrm{HMPAO}$ in primary antiphospholipid antibody syndrome. $\mathrm{J} \mathrm{Nucl}$ Med. 1999;40:1446-1450. 